\title{
A COMPARISON OF THE EFFECTS OF SNOEZELEN AND REMINISCENCE THERAPY ON THE AGITATED BEHAVIOUR OF PATIENTS WITH DEMENTIA
}

\section{Short title}

The effects of Snoezelen on agitation in dementia

\section{Authors}

Sarah BAILLON ${ }^{1}$, Research Associate

Erik VAN DIEPEN ${ }^{2}$, Consultant Psychiatrist

Richard PRETTYMAN² , Consultant Psychiatrist and Honorary Senior Lecturer

Julie REDMAN ${ }^{2}$, Practice Development Nurse and Ward Manager

Nan ROOKE ${ }^{2}$, Practice Development Nurse and Ward Manager

Rowena CAMPBELL ${ }^{2}$, Senior II Occupational Therapist

${ }^{1}$ Department of Health Sciences, University of Leicester

${ }^{2}$ Directorate of Mental Health Services for Older People, Leicestershire Partnership NHS Trust

\section{Corresponding Author}

Sarah Baillon, Psychiatry for the Elderly, University of Leicester, Leicester General Hospital, Gwendolen Road, Leicester, LE5 4PW

Tel.01162584597Ｆax.01162731115Ｅmail.sfb5@le.ac.uk

\section{Sources of research funding}

There was no external funding for this study. It was supported through the facilities and staffing of the Leicestershire Partnership NHS Trust and the University of Leicester.

\section{Key points}

- Both Snoezelen and Reminiscence appear to have some short-term beneficial effect on mood and behaviour, with considerable variation between individual subjects.

- This study did not demonstrate any significant differences between the interventions in terms of impact on subjects' behaviour. 


\title{
A COMPARISON OF THE EFFECTS OF SNOEZELEN AND REMINISCENCE THERAPY ON THE AGITATED BEHAVIOUR OF PATIENTS WITH DEMENTIA
}

\begin{abstract}
Background - Behavioural disturbance, such as agitation, is a common feature of dementia, and causes significant problems and distress for carers. Snoezelen is increasingly used with people who have dementia, but there is limited evidence of its efficacy.
\end{abstract}

Objective - This crossover randomised controlled study aimed to evaluate the effect of Snoezelen on the mood and behaviour of patients with dementia, in comparison to the effect of an established and accepted intervention, reminiscence therapy.

Methods - Twenty patients with dementia and significant agitated behaviour, received three sessions each of Snoezelen and reminiscence. The effects were assessed using measures of observed agitated behaviour and heart rate over the course of the sessions, and mood and behaviour during the sessions.

Results - Both interventions had a positive effect. Snoezelen was no more beneficial than reminiscence in terms of effecting a significant reduction in agitated behaviour or heart rate. There was considerable variation in the way individuals responded to each intervention. Snoezelen may have a more positive effect than reminiscence, but due to the observed differences between the interventions being small, and the small number of subjects, this advantage was not demonstrated statistically. 
Conclusions - Further research, with larger numbers of subjects, and an appropriate control is required to establish the benefits of Snoezelen for people at different stages of dementia, and to identify any benefits additional to those derived from increased staff attention.

\section{Keywords}

Dementia; Therapy; Behavioural symptoms; Agitation, psychomotor.

\section{Introduction}

Agitated behaviour is reported to occur in up to $60 \%$ of patients with $\mathrm{AD}$ (Rabins et al., 1982), and is strongly associated with carer stress and the likelihood of institutionalisation. Because pharmacological treatments have limitations in the treatment of behavioural and psychological symptoms in dementia alternative treatments such as reality orientation, reminiscence and relaxation are of increasing interest. Unfortunately these interventions lack a strong evidence base.

Snoezelen, or multi-sensory environments, originated in the 1960s in the Netherlands in the field of learning disabilities. It is an activity which usually takes place in a dedicated room in which patients may experience a range of unpatterned visual, auditory, olfactory and tactile stimuli (Baker et al., 1997). Snoezelen provides stimulation via the senses of touch, sight, hearing, smell and taste as well as providing vestibular and proprioceptive stimulation as the patient explores the environment. Its aim is to be a relaxing activity, designed "to create a feeling of safety, novelty and stimulation which is under the user's control” (Ashby et al., 1995), and in which there are no expectations for performance. 
Some studies have indicated that Snoezelen can have a positive effect on the mood of people with dementia, may increase patients’ attentiveness to their environment (Moffat et al., 1993; Baker et al., 2001; Spaull et al., 1998), increase appropriate communication (Baker et al., 2001), improve well-being (Sansom et al., 2002) and reduce the occurrence of socially disturbed and challenging behaviour (Johnstone and Finnegan, 2000; Spaull et al., 1998; Kragt et al., 1997; McDonald, 2002). However out of all of the above studies only four (Pinkney, 1997; Baker et al., 2001; Sansom et al., 2002; McDonald, 2002) included an appropriate control condition and only one (Baker et al., 2001) included a suitable number of subjects.

The present research was designed to investigate the value of Snoezelen for treating agitation in people with dementia. This study therefore aimed to compare the effects of Snoezelen and reminiscence therapy on agitated behaviour. Themed reminiscence was selected as the control intervention as it was already accepted as being appropriate for people at all stages of dementia (Woods and Holden, 1995; Finnema et al., 2000), and already used in the clinical areas where the research was taking place. It can also be easily carried out on a one-to-one basis and for a similar duration as the Snoezelen sessions.

\section{Method}

\section{Setting}

This research was carried out at the Bennion Centre, Glenfield General Hospital, at Foxton Grange, which is a charity-run nursing home for older people, and at the Evington Centre, Leicester General Hospital. Both the Bennion and Evington Centres are purpose-built units providing in-patient and day care facilities for the care of older people with mental health 
problems. The Snoezelen rooms at each setting were specifically designed and equipped for multi-sensory intervention and contained a similar range of equipment.

\section{Subjects}

Subjects were included if they had a clinical diagnosis of dementia, were rated by staff as exhibiting behaviour disturbance sufficient to require active intervention as part of the subject's care plan, were available to participate in the research at least two days a week and were judged by staff to be likely to tolerate both types of intervention

Subjects were excluded if they had a pacemaker, had a significant hearing impairment, had significant sight impairment or were non-English speaking. Any subject developing evidence of delirium or having any change in their usual psychotropic or cardiovascular medication, immediately before or during the trial, were withdrawn.

\section{Procedure}

This research was approved by the Leicestershire Health Authority Research Ethics Committee. Potential subjects were identified by the day unit, ward or nursing home manager. If a subject was unable to give informed consent themselves, written agreement was obtained from their next of kin. Using data from a pilot study (van Diepen et al., 2002) it was expected that a sample size of 16 would allow the detection of a difference between the interventions of 3 points in change in observed agitated behaviour (rated on the Agitation Behaviour Mapping Instrument), with a power of $80 \%$ and 0.05 significance.

The study was a randomised controlled cross-over design (Figure 1). Subjects were randomised to one of two groups using a sealed envelope technique. Each subject was 
allocated one of 3 research staff with whom they had all their intervention sessions, this member of staff spent time familiarising themselves with the subject prior to commencing the interventions.

\section{Interventions}

All subjects were required to have 3 one-to-one sessions of each intervention, over the course of a 2 week period, with at least one day between sessions. Sessions lasted up to 40 minutes, unless the subject expressed a desire to leave in which case the session ended immediately. Although the structure of sessions depended upon the individual subject, the content of both Snoezelen and reminiscence sessions were according to guidelines to ensure that the different interventions retained those features that make them distinct from the other and were representative of the way such sessions are carried out during normal clinical practice.

\section{Measures}

At baseline the Mini-Mental State Examination (Folstein et al., 1975) was used to assess cognitive impairment, and the Clinical Dementia Rating Scale (Hughes et al., 1982) was used to rate dementia severity using the 'Sum-of-Boxes' scoring method (Berg et al., 1988). The Cohen-Mansfield Agitation Inventory short form (14 items) (Cohen-Mansfield et al., 1989a) was completed by the subject's keyworker to assess the frequency of agitated behaviour at the care unit.

Agitation Behaviour Mapping Instrument (Cohen-Mansfield et al., 1989b; Cohen-Mansfield, 1986) - The subject's behaviour was observed and rated using the ABMI with reference to 3minute samples before, immediately after, 15 minutes after and 30 minutes after each therapy session. The scale was scored by allocating 1 point for each discrete occurrence of an 
agitated behaviour and 10 points for a continuously agitated behaviour. Inter-rater agreement based on ratings of 6 non-participating patients was good (coefficient of agreement $>0.975$ ). This was the primary outcome variable of the study.

Interact Scale (Baker and Dowling, 1995) - The subjects' behaviour during each session were rated immediately after the session by the researcher who took the session, using the Interact scale. The Interact is a rating scale developed specifically for evaluating the effects of Snoezelen in dementia care. The scale comprises items relating to the mood and behaviour of a patient, which are rated on a 5 point scale. A revised version of the Interact was used in this study. This was based upon the 12-item short form of the scale (Baker and Dowling, 1995), with one additional item ('spoke sensibly') from the longer version of the scale. Each item was scored for direction of change. As the scale does not give a total score these modifications did not require further analysis.

Heart Rate - Subjects' heart-rate (beats per minute) was recorded at 1-minute intervals from approximately 15 minutes before, until 30 minutes after the sessions using a heart-rate monitor (CardioSport 2001). Heart rate and the Interact scale were the secondary outcome variables of the study.

\section{Analysis}

The data were analysed using SPSS for Windows, according to the method described by Altman (Altman, 1999) for the analysis of crossover trials. Non-parametric tests were used throughout the analysis of data due to the small sample involved, and the non-normal distribution of the variables being analysed. Summary variables were calculated for the analysis of repeated measures (Matthews et al., 1990). Confidence intervals (CI) for the 
difference between the medians were calculated according to the methods described by Altman et al. (2000).

\section{Results}

A total of 20 subjects completed the study protocol - 5 were recruited from the Bennion Centre, 10 from Foxton Grange Nursing Home, 5 from the Evington Centre. 5 subjects were randomised but did not complete the study protocol (see Table 1). 1 subject, although appearing to enjoy the introductory Snoezelen session at the time, became distressed upon recalling the room, and so dropped out following 2 sessions of reminiscence. 2 subjects were admitted to residential care, 1 subject refused to leave the lounge for the research sessions and 1 suffered a stroke during the wash out period, and was unable to continue. No subjects were excluded due to change in their medication.

\section{Observed agitated behaviour}

The frequency of agitated behaviour was observed at four different time points. Figure 2 represents the mean ABMI scores for the whole study group before and after the research sessions. Table 2 reports the difference between pre-session and immediately post-session, and pre-session and 15 minutes post session in ABMI scores for each intervention.

There was no statistically significant difference between Snoezelen and Reminiscence sessions in terms of the change in level of agitation from pre-session to immediately postsession (Wilcoxon Signed Rank Test $\mathrm{Z}=-1.33, \mathrm{P}=0.18,95.9 \% \mathrm{CI}-4.3$ to 2.0 ), nor from presession to 15 minutes after the sessions (Wilcoxon Signed Rank Test $\mathrm{Z}=-0.16, \mathrm{P}=0.87$, 95.9\% CI -2.0 to 3.4$)$. 


\section{Heart Rate}

Heart rate data was available for all except two subjects, who did not tolerate the monitor (Figure 3). One subject had atrial fibrillation and so their data was not included in the analysis. For the remaining 17 subjects, data was missing for 5 Snoezelen and 3 reminiscence sessions (7 different subjects) due to data recording failure.

Heart rate data was analysed by calculating the difference between the mean heart rate for a 5-minute period before the research session (3-8 minutes before), 5 minutes before the end of the research session (excluding the final 2 minutes) and 5 minutes after the research session (3-8 minutes after) for each intervention (see Table 2).

There was no significant difference between the two interventions in terms of change in mean heart rate during the session (Mann-Whitney $\mathrm{U}=28.0, \mathrm{P}=0.50,95.7 \% \mathrm{CI}-7.49$ to 4.47 ), nor after the session (Mann-Whitney=23.0, $\mathrm{P}=0.24,95.7 \% \mathrm{CI}-11.23$ to 5.53).

\section{Interact Scale}

The mean number of items rated as showing positive and negative were compared between the interventions (see Table 2). There was no significant difference between the two interventions in terms of the number of items rated as showing positive change during the session (Wilcoxon Signed Rank Test $\mathrm{Z}=-1.61, \mathrm{P}=0.11$, 95.9\% CI 0 to 2.7) nor negative change during the session (Wilcoxon Signed Rank Test $\mathrm{Z}=-0.15, \mathrm{P}=0.88,95.9 \% \mathrm{CI}-0.3$ to $0)$. 


\section{Discussion}

This research has not shown that Snoezelen is more beneficial for people with dementia than one-to-one themed reminiscence. The high numbers of Interact items rated as showing improvement indicate that both reminiscence and Snoezelen have a positive effect on the mood and behaviour in this patient group. The observed differences between the interventions were small for change in ABMI and heart rate, and with the small numbers involved in this study, it may mean that genuine differences were not demonstrated. There was substantial variation between the subjects in their response to sessions and when the data were combined the groupwise differences between interventions were modest.

The specific focus of this research was regarding the effect of Snoezelen on agitated behaviour. Data from this study is inconclusive. Snoezelen may have a calming effect on some patients who are agitated, but patients' responses appear to be variable. In this study sample some patients appeared to be benefiting from Snoezelen or reminiscence, whereas for other patients the interventions did not have a substantial effect on agitation. This is probably one of the reasons that the observed group differences for the ABMI and heart rate were nonsignificant.

\section{Methodological issues}

Each subject had the same 'therapist' for both interventions, so the study is controlled for intervention differences due to different staff. This therefore enabled the evaluation of the effects of Snoezelen independent of the positive effects derived from increased staff attention. The study design did not allow for the researchers and keyworkers being blind to the intervention the subjects' were receiving. 
This research has indicated that both Snoezelen and themed reminiscence can have beneficial effects for some, but not all people with dementia, including those with severe dementia. It has added to the increasing evidence that Snoezelen can at the very least, be an enjoyable and positive activity for people with dementia, and offers additional choice of an intervention that is appropriate for such patients, where many other activities are unsuitable.

In addition to any direct benefits of the intervention, Snoezelen provides an ideal opportunity for staff to focus on the 'experience' and sensory needs of patients in their care, has benefits for staff-patient relationships, and appears to legitimise spending time away from the ward and spending ‘quality time’ with patients (Ellis and Thorn, 2000).

The impact of Snoezelen on behaviour, and the longevity of any effect, over and above that of a control intervention, remains inconclusive (although some individuals do show marked benefit). Further well-designed empirical research, which includes sufficient patient numbers, and suitable control interventions, is needed to establish this. It may be that Snoezelen is not able to produce a lasting effect on behaviour or mood for people with dementia. However it does appear that Snoezelen can achieve at least a "time-limited benefit” (Hogg et al., 2001) for people with severe dementia who can no longer benefit from many other approaches. 


\section{Acknowledgement}

This research would not have been possible without the help and support of the patients and their carers, nor without the help of the staff at Foxton Grange Nursing Home, the Bennion Centre and the Evington Centre.

Snoezelen is a registered trademark of ROMPA International.

\section{References}

Altman, D.G. 1999. Practical statistics for medical research. Chapman and Hall/CRC, London.

Altman, D.G., Machin, D., Bryant, T.N., Gardner, M.J. 2000. Statistics with confidence. BMJ Books.

Ashby, M., Lindsay, W.R., Pitcaithly, D., Broxholme, S. and Geelan, N. 1995. Snoezelen: its effects on concentration and responsiveness in people with profound multiple handicaps. British Journal of Occupational Therapy, 58(7): 303-307.

Baker, R. and Dowling, Z. 1995. Interact. Dorset Healthcare NHS Trust.

Baker, R., Bell, S., Baker, E. et al. 2001. A randomised controlled trial of the effects of multisensory stimulation (MSS) for people with dementia. British Journal of Occupational Therapy 40: 81-96.

Baker, R., Dowling, Z., Wareing, L.A., Dawson, J. and Assey, J. 1997. Snoezelen: its longterm and short-term effects on older people with dementia. British Journal of Occupational Therapy 60(5): 213-218.

Berg, L., Miller, J.P., Storandt, M. et al. 1988. Mild dementia of the Alzheimer type: 2. Longitudinal assessment. Annals of Neurology 23: 477-484. 
Cohen-Mansfield, J. 1986. Guidelines and suggestions for administering the agitation behaviour mapping instrument (ABMI). Research Institute of the Hebrew Home of Greater Washington, Rockville, MD, USA.

Cohen-Mansfield, J., Marx, M.S. and Rosenthal, A.S. 1989a. A description of agitation in a nursing home. Journal of Gerontology 44: M77- M84.

Cohen-Mansfield, J., Werner, P. and Marx, M.S. 1989b. An observational study of agitation in agitated nursing home residents. International Psychogeriatrics 1(2): 151-165.

Ellis, J. and Thorn, T. 2000. Sensory stimulation: where do we go from here? Journal of Dementia Care 8(1): 33-37.

Finnema, E., Droes, R.M., Ribbe, M. and van Tilburg, W. 2000. The effects of emotionoriented approaches in the care for persons suffering from dementia: A review of the literature. International Journal of Geriatric Psychiatry 15: 141-161.

Folstein, M., Folstein, S. and McHugh, P. 1975. Mini-Mental State - a practical method for grading the cognitive state of patients for the clinician. Journal of Psychiatric Research, 12: 189-198.

Hogg, J. Cavet, J., Lambe, L. and Smeddle, M. 2001. The use of Snoezelen as multisensory stimulation with people with intellectual disabilities: a review of the literature. Research in Developmental Disabilities 22(5): 353-372.

Hughes, C.P., Berg, L., Danziger, W.L., Coben, L.A. and Martin, R. 1982. A new clinical scale for the staging of dementia. British Journal of Psychiatry 140: 566-572.

Johnstone, S. and Finnegan, M. 2000. Sights and Sounds. Therapy Weekly 26(49): 55. 
Kragt, K., Holtkamp, C.C.M., Van Dongen, M.C.J.M., van Rossum, E. and Salentijn, C. 1997. Het effect van snoezelen in de snoezelruimte op het welbevinden van demente ouderen. Verpleegkunde 12(4): 227-236.

Matthews, J.N.S., Altman, D.G., Campbell, M.J. and Royston, P. 1990. Analysis of serial measurements in medical research. British Medical Journal 300: 230-235.

McDonald, C. 2002. Back to the real sensory world our 'care' has taken away. Journal of Dementia Care 10(1): 33-36.

Moffat, N., Barker, P., Pinkney, L., Garside, M. and Freeman, C. 1993. Snoezelen. An experience for people with dementia. Dorset HealthCare NHS Trust.

Pinkney, L. 1997. A comparison of the Snoezelen environment and a music relaxation group on the mood and behaviour of patients with senile dementia. British Journal of Occupational Therapy, 60(5), 209-212.

Rabins, P.V., Mace, N.L. and Lucas, M.J. 1982. The impact of dementia on the family. Journal of the American Medical Association, 248, 333-335.

Sansom, G., Coleman, M., Young, I., Pope, P. and Richards, C. 2002. Multi-sensory therapy versus Reminiscence therapy. Signpost, 7(1), 7-10.

Spaull, D., Leach, C. and Frampton, I. 1998. An evaluation of the effcets of sensory stimulation with people who have dementia. Behavioural and Cognitive Psychotherapy, 26, 77-86.

van Diepen, E., Baillon, S., Redman, J., Rooke, N., Spencer, D. and Prettyman, R. 2002. A pilot study of the physiological and behavioural effects of Snoezelen in dementia. British Journal of Occupational Therapy, 65(2), 61- 66. 
Woods, R.T. and Holden, U. 1995. Positive approaches to dementia care. Churchill Livingstone. 
Table 1 Gender, age, CDR, MMSE, CMAI (Median, (inter-quartile range)) and diagnosis of subjects

\begin{tabular}{lll}
\hline & Subjects who completed study & Subjects who dropped out \\
\hline Gender & 8 male, 12 female & 3 male, 2 female \\
Age (yrs) & $73.5(63.7,81.8)$ & $80.0(72.0,82.5)$ \\
CDR & $17.0(15.0,18.0)$ & $17.0(12.0,17.0)$ \\
MMSE & $4.0(0,9.0)$ & $1.0(0,11.5)$ \\
CMAI & $25.5(21.3,30.3)$ & $27.0(24.0,36.5)$ \\
Diagnosis* & $12-$ Alzheimer’s disease & $1-$ Alzheimer's disease \\
& $6-$ Vascular dementia & $1-$ Vascular dementia \\
& $1-$ Alcohol-related dementia & $1-$ mixed dementia \\
& $1-$ dementia - unspecifed & 2 - dementia - unspecifed \\
\hline
\end{tabular}

* Clinical diagnosis as assigned by subject's consultant psychiatrist in their notes 
Table 2 Results of measures for each intervention (Median (IQR))

SNOEZELEN REMINISCENCE

\section{ABMI change*}

Pre- to Post-session

$-1.3(-3.3,3.0) \quad 0.8(-1.6,3.3)$

Pre- to 15-mins after session

$0.7(-2.6,2.6) \quad-0.2(-4.7,2.8)$

\section{Heart rate change*}

Pre- to during session

$-1.5(-5.8,1.2) \quad-1.4(-4.2,2.1)$

Pre- to after session

$-1.6(-4.8,1.6)$

$1.1(-4.8,3.8)$

\section{Interact}

Mean number of items with positive change

$4.3(1.0,6.5)$

$2.5(0.1,6.0)$

Mean number of items with negative change

$0(0,0.9)$

$0(0,1.3)$

* A negative value indicates a decrease. 
Figure 1 Cross-over design

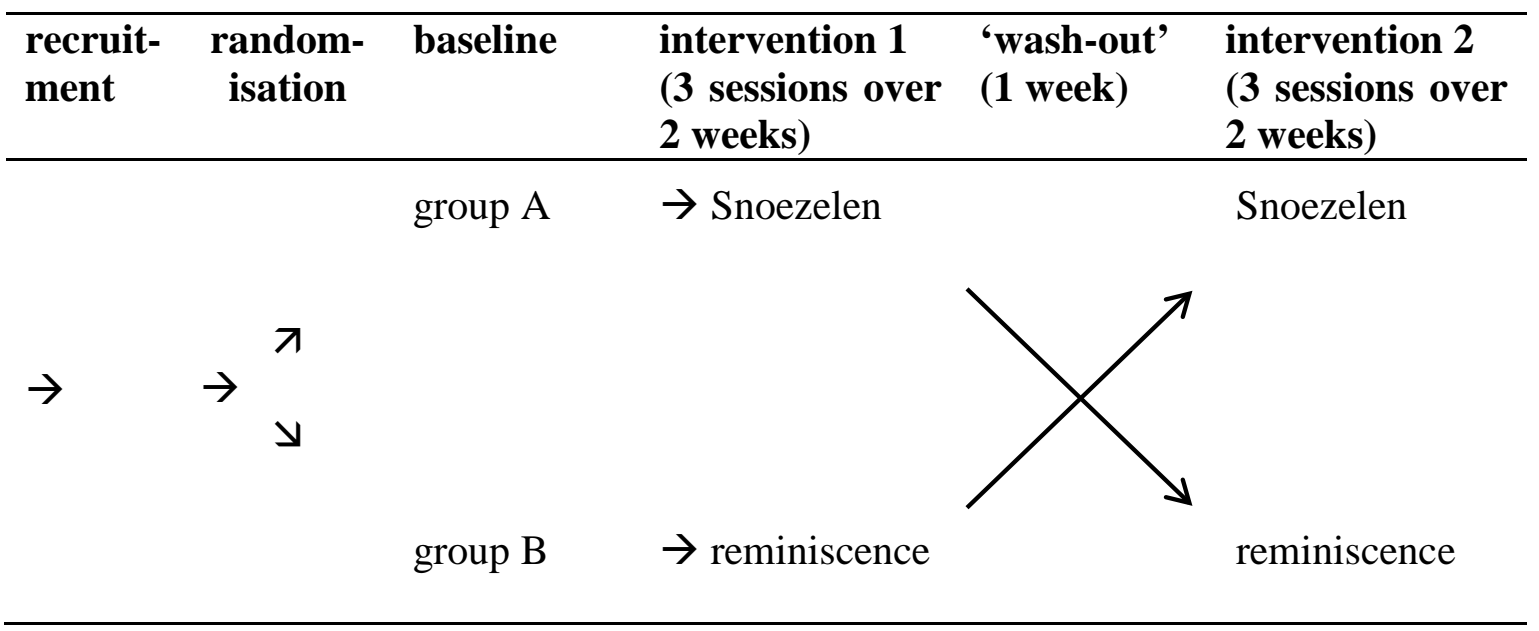


Figure 2 Mean ABMI score before and after sessions

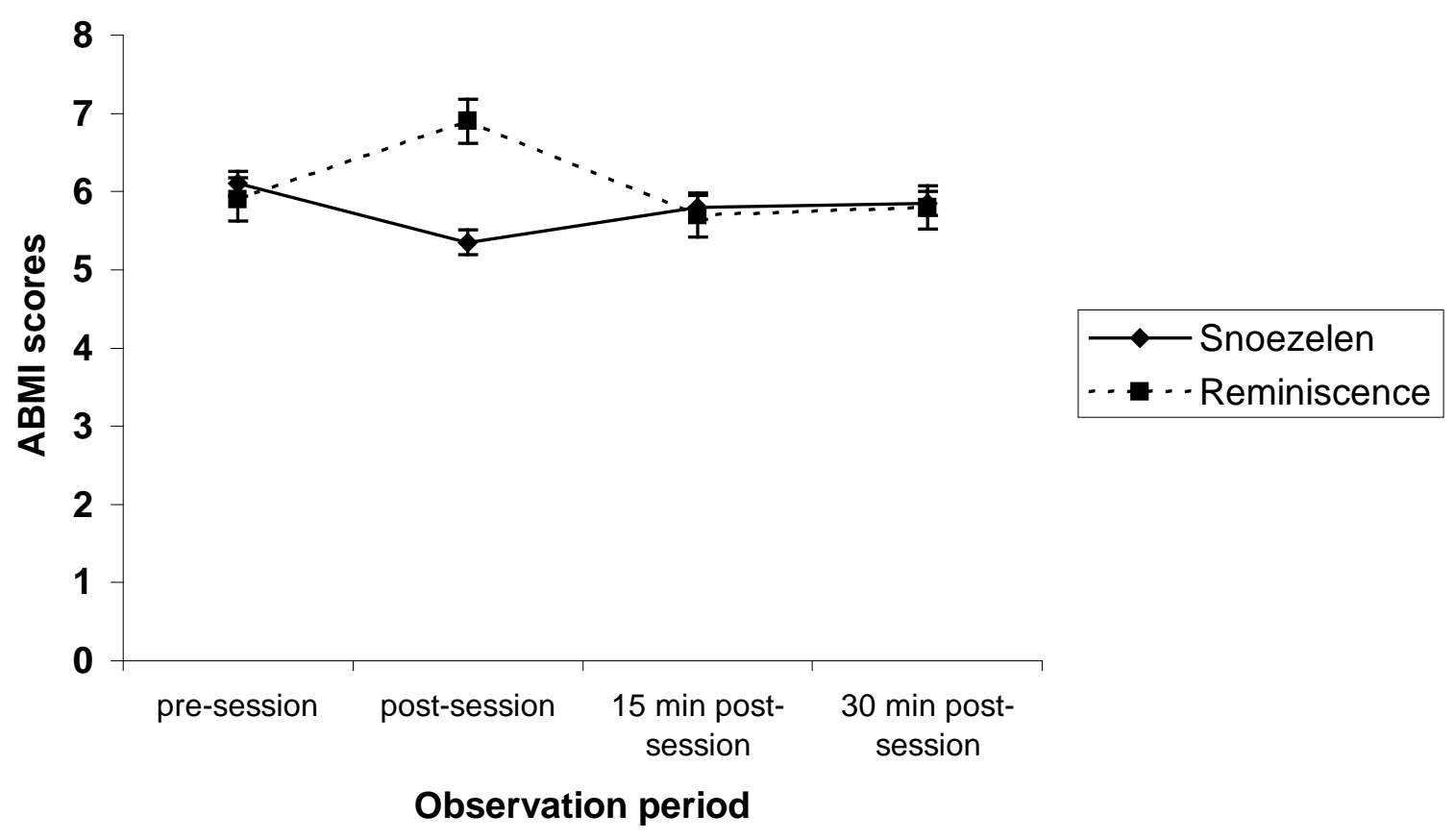


Figure 3 Mean heart rate for all subjects

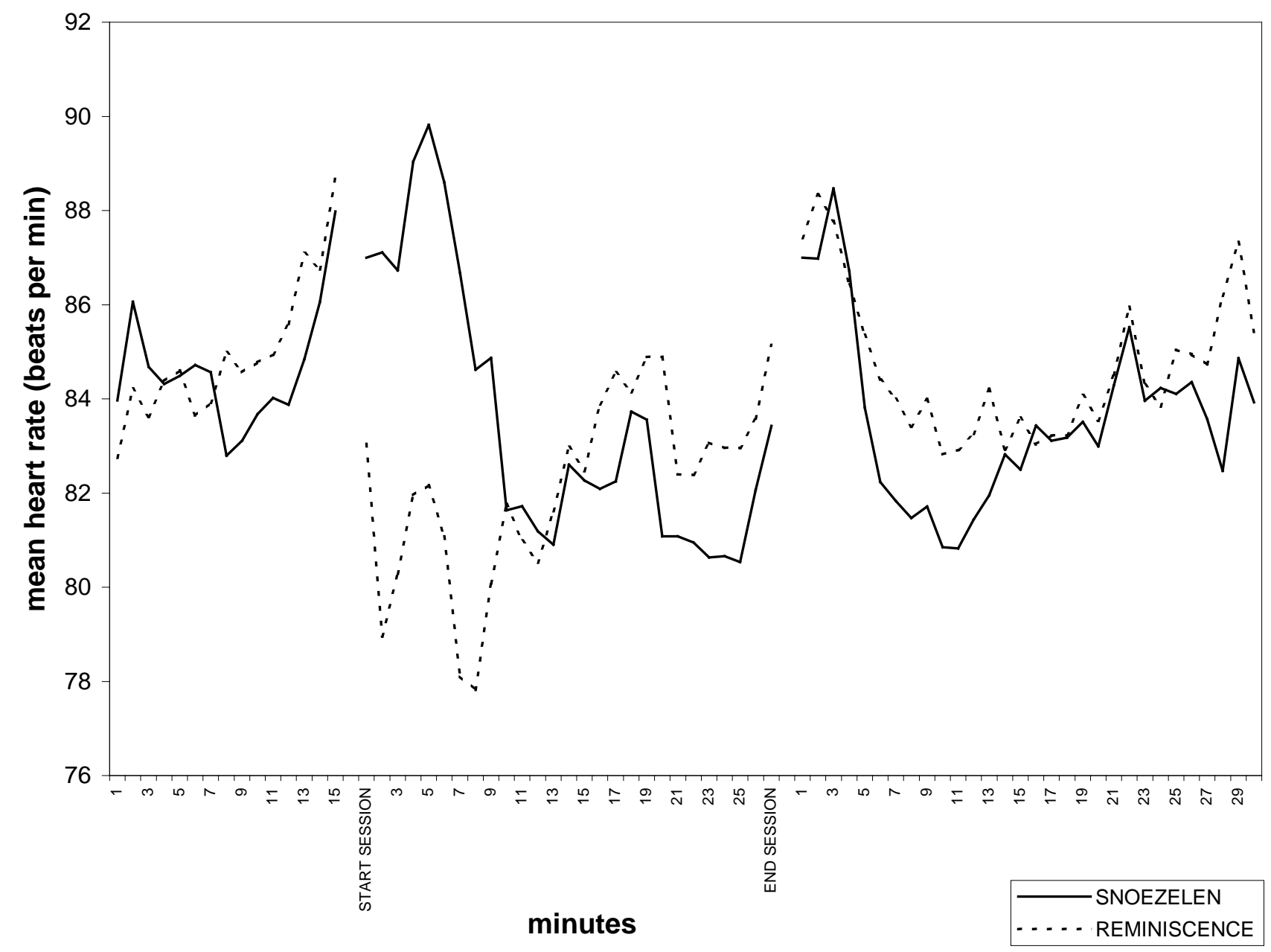

